\title{
Rosmarinic acid in Canna generalis activates the medial deterrent chemosensory neurone and deters feeding in the tobacco hornworm Manduca sexta
}

\author{
MONIQUE S. J. S IMMONDS ${ }^{1}$, PHILIP C. STEVENSON ${ }^{1}$ \\ and F R A K E. H A N O N ${ }^{2}$ \\ ${ }^{1}$ Royal Botanic Gardens, Kew, Surrey, U.K. and ${ }^{2}$ Department Biological Sciences, University of Maryland Baltimore County, \\ Baltimore, Maryland, U.S.A.
}

\begin{abstract}
For all but a very few highly specialized insect feeders, Canna generalis L. (Cannaceae) is unacceptable as a food plant and is a highly potent feeding deterrent for the tobacco hornworm Manduca sexta Johan. (Lepidoptera). The present study describes the isolation of an active deterrent compound, rosmarinic acid (RA) from the ethanol extract of canna leaves. A two-choice bioassay shows that RA is a feeding deterrent in the concentration range $0.3-3 \mathrm{~mm}$. Bilateral ablation of the oral chemoreceptors eliminates this deterrence. The results reported in the behavioural literature show that only the epipharyngeal sensilla and the medial (but not the lateral) styloconica contribute to this deterrence elicited by canna foliage or extract, and the data obtained in the present study show the same results using RA. Electrophysiological recordings from the medial styloconica show that RA primarily stimulates the 'deterrent neurone' in a concentration-dependent manner with a threshold of approximately $0.03 \mathrm{~mm}$ and a peak frequency of 69 spikes s $^{-1}$ at 0.1 mM RA. An extract of canna leaves elicits similar responses. Adding RA to canna extract does not elicit another class of spikes, indicating that both stimuli activate the same neurone. We discuss the significance of the results with respect to furthering our knowledge about the sensory basis of food selection, and specifically about the range of compounds that stimulate deterrent chemosensitive neurones of lepidopteran larvae.
\end{abstract}

Key words. Feeding behaviour, insect neurophysiology, Lepidoptera, phagodeterrents, phytochemistry, sensory coding.

\section{Introduction}

Diet specialization and the selection of food are important aspects of insect life strategies (Simpson \& Raubenheimer, 1993). Such selectivity requires physiological systems to recognize potential food and nonfood. For phytophagous insects, this means that the detection capabilities of the sense organs must match the compounds and their concentrations in the encountered plants. Many phytophagous insects are highly selective in their feeding habits, and so phagodeterrence mechanisms likely play an important role in feeding decisions in these insects (Chapman, 1995). Accordingly, our understanding of

Correspondence: Frank Hanson, Department of Biological Sciences, University of Maryland Baltimore County, Baltimore, Maryland 21250, U.S.A. Tel.: 410-455-2261; e-mail: hanson@umbc.edu food selection depends on our knowledge of the deterrent phytochemicals, the sensory mechanisms by which they are detected and the central nervous system processes that interpret their signals to be a phagodeterrent (Chapman, 1995).

One plant that is rarely damaged by insects is canna (Canna generalis L., Cannaceae), an ornamental that is commonly grown in public gardens. It is very strongly phagodeterrent for the tobacco hornworm Manduca sexta Johan. (Lepidoptera, Sphingidae). Several behavioural studies using this model insect describe employing canna as the nonhost plant (Jermy et al., 1968; de Boer \& Hanson, 1987; Peterson et al., 1993). Canna is such a deterrent that hornworms prefer to starve rather than eat it; however, they will readily feed on it if their taste organs have been removed (de Boer \& Hanson, 1987). Canna does not appear to be poisonous: if a diet of canna mixed with host plant is fed to these chemosensory-ablated caterpillars, no toxic effects 
are observable; most gain weight, pupate and eclose normally. Thus, it is likely that a phytochemical in canna leaves is deterrent but not toxic to $M$. sexta.

The detection of the phagodeterrent in canna is apparently by contact chemoreception, and not olfaction (de Boer \& Hanson, 1987). When hornworms encounter a canna leaf, they take a small test bite and crawl away; removal of all the olfactory organs (antenna and maxillary palps) does not change this. Similarly, the lateral styloconica of the maxilla do not appear to play a role in rejection of canna because their presence or absence makes no difference in these experiments. Removal of the other taste organs, however, has dramatic consequences: ablation of both the medial styloconica of the maxilla and the epipharyngeal sensilla permits larvae to feed on either canna or host plants indiscriminately. Thus, the gustatory receptors in the medial styloconica and epipharyngeal sensilla are clearly the organs responsible for detecting the deterrent(s) in canna.

An active principle in canna leaves can be extracted in hot ethanol (Peterson et al., 1993). A partially purified fraction tested electrophysiologically elicits large spikes of a single type from the medial styloconicum, the frequency of which correlates well with phagodeterrency in a concentration-dependent manner. The conclusion is that these spikes are produced by a deterrent neurone mediating the behavioural rejection of canna. In view of the importance of understanding phagodeterrence and its role in host selection, the present study isolates and identifies a deterrent phytochemical in canna and confirms its activity using behavioural and electrophysiological bioassays.

\section{Materials and methods}

\section{Chemical isolation and identification of rosmarinic acid (RA)}

The procedure described by Peterson et al. (1993) was used to obtain an active crude extract of the foliage of $C$. generalis. Leaves were de-veined, weighed, ground in liquid nitrogen and extracted in boiling $95 \%$ ethanol; this extract was then filtered, defatted by extracting twice with hexane, reduced in volume with a rotary evaporator under reduced pressure, evaporated to dryness under nitrogen and dissolved in $10 \%$ ethanol and $0.1 \mathrm{M}$ $\mathrm{NaCl}$. This stock solution was adjusted to $0.84 \mathrm{~g}$ fresh weight of canna $\mathrm{mL}^{-1}$, and then stored under nitrogen at $-10^{\circ} \mathrm{C}$ in the dark.

Fractionation was carried out by reverse-phase column chromatography that separates polar compounds on the basis of polarity. Three millilitres of the stock solution was concentrated in vacuo at $<30^{\circ} \mathrm{C}$ and the resulting $0.038 \mathrm{~g}$ was dissolved in $0.5 \mathrm{~mL}$ of distilled water. This extract was applied to a Sep-Pak cartridge (Waters, Milford, Massachusetts) that had been previously washed with methanol and then water, and elution was performed sequentially with $3 \mathrm{~mL}$ of water (fraction 1), $50 \%$ methanol (fraction 2) and methanol (fraction 3). Electrophysiological and behavioural assays showed that fraction 2 elicited the greatest response from the deterrent neurone in the medial styloconica (Peterson et al., 1993). Therefore, for the present study, this same fraction 2 was used; it was filtered through
0.45 micron filters (Millipore, Burlington, Massachusetts) and analyzed using a high-performance liquid chromatography (HPLC) system consisting of an LC 600 pump, model 717 autosampler and model 996 photodiode array detector (Waters). Samples were injected onto a Lichrospher 5ODS end-capped column $(25 \mathrm{~cm} \times 4.0 \mathrm{~mm}$ inner diameter) (Millipore) and separated using a binary gradient (solvent $\mathrm{A}=2 \%$ acetic acid in water; solvent $\mathrm{B}=18: 1: 1$ methanol : water : acetic acid) where at $\mathrm{T}=0 \mathrm{~min}, \mathrm{~A}=75 \%$ and at $\mathrm{T}=20 \mathrm{~min}, \mathrm{~A}=0 \%$ using a linear gradient with flow rate of $1.0 \mathrm{~mL} \mathrm{~min}^{-1}$. The analytical method was adapted for preparative HPLC using a Spherisorb 5ODS column $(25 \mathrm{~cm} \times 10 \mathrm{~mm}$ inner diameter) (Waters) and a binary gradient (solvent $\mathrm{A}=2 \%$ acetic acid in water; solvent $\mathrm{B}=2 \%$ acetic acid in methanol) where at $\mathrm{T}=0, \mathrm{~A}=80 \%$ and at $\mathrm{T}=20, \mathrm{~A}=0 \%$ using a linear gradient with flow rate of $4.73 \mathrm{~mL} \mathrm{~min}^{-1}$. Seven subfractions of fraction 2 were collected manually; each subfraction contained either a main peak or a few minor peaks. These fractions were then tested in an electrophysiological bioassay.

Insects

For the first cohort of larvae, eggs of $M$. sexta were obtained from the stock USDA colony at Beltsville (Maryland). These larvae were maintained on an artificial diet modified from Yamamoto (1969) and used for the behavioural concentration-response studies and electrophysiology. For the second cohort of larvae used for chemosensory deprivation experiments, eggs were obtained from Carolina Biological (Burlington, North Carolina) and maintained on a commercial tobacco hornworm media (Bio-Serve, Frenchtown, New Jersey) that is essentially the same as the diet used by Yamamoto (1969). For both cohorts, late fourth-instar premoults were removed from the culture daily and isolated at room temperature. After moulting into the fifth instar and just before testing, each animal was given $1 \mathrm{~h}$ of ad libitum access to six water-saturated filter paper discs to standardize larval hydration. Preliminary experiments showed this procedure to be important: water-deprived animals tolerated some levels of deterrents, presumably to obtain water from the solution.

\section{Behavioural assays}

Behavioural responses of the larvae were determined using the two-choice disk test modified from de Boer \& Hanson (1987). Briefly, discs of $1.5 \mathrm{~cm}$ in diameter were punched from edible glass-fibre filter paper (GF/A; Whatman, U.K.) and pinned into a styrofoam floor at a height of $1 \mathrm{~cm}$. Six discs were arranged in a circle of $10 \mathrm{~cm}$ in outer diameter and covered by the lid of a Petri dish (diameter $12 \mathrm{~cm}$ ) to form a chamber. Three discs treated with $0.1 \mathrm{~mL}$ of test solution were alternated with three control discs wetted with $0.1 \mathrm{~mL}$ of distilled water. The test began when one larva was placed with random orientation in the center of the chamber and allowed to feed until approximately half of the disc area of the most-eaten choice, either treated or control, was consumed. The area that was eaten for all six 
discs was estimated visually to $\pm 5 \%$ or by software analysis of digital photos spot-checked visually and summed within each category (treated or control). An index was computed such that phagodeterrence is positive ( 0 to 100$)$ and phagostimulation negative $(0$ to -100$)$ :

$$
\begin{aligned}
& \text { Deterrence index } \\
& =[(\text { control-treated }) /(\text { control }+ \text { treated })] \times 100
\end{aligned}
$$

Tests were conducted at room temperature (approximately $22^{\circ} \mathrm{C}$ ) using RA (Sigma-Aldrich, St Louis, Missouri) as the treatment. Larvae were tested only once and then discarded.

\section{Chemosensory deprivation}

Early fifth-instar larvae from the second cohort were anaesthetized by immersion in water for 10-20 min (Gothilf \& Hanson, 1994). Control larvae were anaesthetized but not subjected to surgery. Control and experimental larvae were tested at the same time under the same conditions. Bilateral extirpations were performed on the experimental larvae using the techniques described by de Boer \& Hanson (1987) employing small iridectomy scissors for amputations comprising the lateral styloconica of the maxilla; both medial and lateral styloconica by removal of the galea of the maxilla; and epipharyngeal sensilla by removal of the epipharynx. Verification of successful bilateral organ removal without damage to nontarget organs was conducted microscopically after recovery. After an overnight recovery period and the water pre-test described above, one larva was placed into a two-choice disk test with $1 \mathrm{~mm}$ RA (ICN, Costa Mesa, California) on the treatment discs and distilled water on the control discs. The significance of differences in responses between groups was determined by a nonparametric Kruskal-Wallace test $($ alpha $=0.05)$.

\section{Electrophysiology}

The intact animal preparation of Gothilf \& Hanson (1994) was used for the electrophysiological recordings. An anaesthetized larva was inserted backwards into a glass vial with only its head protruding through a hole in a thin rubber gasket and the vial cap. The vial contained a $\mathrm{Ag}-\mathrm{AgCl}$ wire reference electrode and $0.1 \mathrm{M} \mathrm{KCl}$ that provided electrical connection between this reference electrode and the larval body surface. In addition, the maintained immersion suppressed the animal's movements throughout the approximately 4-h experiment without affecting the chemosensory responses. At the end of the experiment, the animal was released from the vial and observed to be mobile and feeding the next day. Each animal was used only once.

Neural responses of the styloconica were obtained electrophysiologically using the standard tip recording technique (Frazier \& Hanson, 1986; Gothilf \& Hanson, 1994). A micropipette with a tip diameter of approximately $50 \mu \mathrm{m}$ containing the stimulus was placed over the tip of a maxillary styloconicum, allowing the stimulus solution to contact the sensory dendrite through the apical pore. The micropipette also contained an electrolyte $(0.1 \mathrm{M} \mathrm{KCl})$ in contact with a $\mathrm{Ag}-\mathrm{AgCl}$ surface that served as the active electrode. This was connected to a high impedance preamplifier (George Johnson, Baltimore, Maryland) (Frazier \& Hanson, 1986) with a rapid baseline-restoring circuit that minimized contact artefacts and permitted undistorted action potentials to be recorded within $10 \mathrm{~ms}$ after contact. The signal was amplified with a bandpass of $100 \mathrm{~Hz}$ to $3 \mathrm{kHz}$ and saved in digital format using data acquisition software ('STA'; E. Städler, Federal Research Station, Switzerland) sampling at $10 \mathrm{kHz}$. A single trial consisted of sequentially contacting the tip of each lateral and medial styloconicum bilaterally with the stimulating/recording micropipette for $1.5 \mathrm{~s}$. Three of these trials were conducted with each stimulus; inter-trial intervals of $3 \mathrm{~min}$ permitted full recovery of the sensory neurones between trials. A $0.1 \mathrm{M} \mathrm{KCl}$ control trial was performed at the beginning and end of the experiment and after each change of stimulus for a total of nine or more control trials. Action potentials were classified and counted for $1 \mathrm{~s}$ with custom software using digital filtering to isolate spikes and align their peaks, and then a template matching procedure was used to sort them (Stitt et al., 1998; as modified by M. Rowley, Louisville, Kentucky). Classification was spot-checked manually. We recorded from eight larvae, all of which provided similar responses. From these, we analyzed data from six medial and lateral styloconica pairs that provided good recordings from both styloconica and signal-to-noise ratios suitable for spike classification.

Solutions of pure chemicals used for electrophysiological testing were RA (Sigma-Aldrich Chemicals) and caffeine (Sigma-Aldrich) dissolved in $0.1 \mathrm{M} \mathrm{KCl}$. Canna stimuli were obtained from the ethanol extract of canna leaves adjusted to the original leaf concentration (reported as ' $1 \times$ ', as in Peterson et al., 1993). Dilutions of the $1 \times$ were caried out with $0.1 \mathrm{M}$ $\mathrm{KCl}$ to maintain electrolyte concentration. Mixtures of RA and canna extract were made by mixing equal volumes of selected RA concentrations and canna extract dilutions. Thus, if each solution contained the same chemical, its concentration in the mixture would be maintained, whereas the concentration of dissimilar chemicals would be halved.

\section{Results}

\section{Identification of a phagodeterrent chemical in canna}

HPLC analysis of the biologically active $50 \%$ methanol fraction (fraction 2) of the crude ethanol extract of canna revealed a major peak at $14.52 \mathrm{~min}$. This peak and other minor peaks were collected by HPLC into seven subfractions, each of which was assayed for activity by electrophysiology. The most active subfraction contained a peak that was identified as RA (Fig. 1), an ester of caffeic acid and 3,4-dihydroxyphenyllactic acid. The identification of RA was confirmed by co-chromatography and ultraviolet-spectral comparison with an authentic standard (Apin Chemicals, U.K.). RA was present at $1.6 \mathrm{mg} \mathrm{g}^{-1}$ fresh weight of foliage ( $4.4 \mathrm{~mm}$, assuming all fresh weight is active solution) 
and constituted $85 \%$ of the ultraviolet-absorbing components in the $50 \%$ methanol fraction.

\section{Behavioural responses to $R A$}

RA is clearly a strong phagodeterrent for hornworms; the concentration-response curve of two-choice bioassays of RA versus water showed that the deterrence threshold is approximately $0.3 \mathrm{~mm} \mathrm{RA}$ (the concentration at which deterrence by RA is significantly greater than that of water, $P=0.025, t$-test). Deterrence is almost complete at $1 \mathrm{~mm}$ RA (Fig. 2).

Differential ablation experiments provide an indication of the sensory pathway of deterrence by RA. First, removing only the lateral styloconica did not significantly reduce aversion to RA [lateral (Lat) versus not ablated (None), $P=0.746$, Kruskal-Wallace test] (Fig. 3). This indicates that the laterals are not a major contributor to the RA deterrence pathway, and that 'Lat' may also be regarded as a sham control for these experiments. Second, when the medial styloconica were also removed, deterrence was significantly reduced compared with larvae that still possessed them [lateral and medial (ML) and epipharynx, medial and lateral (EML) versus None, Lat and epipharyngeal sensilla (Epi), $P<0.0001$, Kruskal-Wallace) (Fig. 3). Third, larvae with lateral and medial ablations were significantly less deterred than those with only laterals ablated (ML versus Lat, $P=0.044$, Kruskal-Wallace) (Fig. 3); because the only difference here is the absence of the medial, the evidence indicates that it is the primary organ mediating aversion to RA.

A caveat here is that the concentration-response experiments were performed with a different cohort of larvae than those used in the ablation experiments. However, both cohorts show strong deterrence at the same concentration of RA (1 mM) (Fig. 2 versus Fig. 3, None), suggesting that there was little if any difference between these cohorts.

\section{Chemosensory neurone responses}

Responses to $0.1 \mathrm{~m} \mathrm{KCl}$. Because all electrophysiological stimuli contained $0.1 \mathrm{M} \mathrm{KCl}$, this was used as the control stimulus for the electrophysiological recordings. It elicited low frequency responses (approximately $20 \mathrm{~s}^{-1}$ ) of two neurones in both the medial and lateral styloconica (Fig. 4, trace 1; Fig. 5 at 0 concentration of RA and canna). These responses remained constant in the nine or more control trials throughout the $4 \mathrm{~h}$ experiment.

Responses to RA. Responses from the medial styloconica to RA consisted mainly of large spikes of uniform shape and inter-spike interval, indicating a single neurone source (Fig. 4A, traces 2 and 3). The activity of this 'RA neurone' was concentration-dependent, with a threshold of $<0.03 \mathrm{~mm}$ and mean peak frequency of $69 \pm 7$ spikes $\mathrm{s}^{-1}$ at $0.1-1 \mathrm{~mm}$ (Fig. 5A, solid lines and symbols). A second, smaller spike was also seen (Fig. 4A, trace 2) that decreased in frequency at higher RA concentrations (Fig. 5A, open symbols). These might be responses of the 'salt neurone' to the $0.1 \mathrm{M} \mathrm{KCl}$ in the stimulus solution.

Responses to RA from the lateral styloconica (Fig. 4B, traces 2 and 3 ) are similar to those of the medial styloconica, although with somewhat higher spike frequencies of the small spike (Fig. 5A). Interestingly, the average frequencies of the large spikes of the deterrent neurones were very similar in the two styloconica (Fig. 5A).

Responses to canna extract. Responses from the medial styloconica elicited by canna extract (Fig. 4A, traces 4 and 5) were very similar to those elicited by RA (Fig. 4A, traces 2 and 3). Responses from the lateral styloconica to canna (Fig. 4B, traces 4 and 5) appeared to be more heterogeneous than those of the medial styloconica (Fig. 4A, traces 4 and 5), presumably as a result of more types of sensory neurones firing. As with the RA stimuli, the average frequencies of the large spikes were comparable in the two styloconica (Fig. 5B).

Responses to mixtures of RA and canna extract. Adding RA to the canna stimulus did not elicit a new class of spikes from the medial styloconica, comprising evidence that the neurone responding to $\mathrm{RA}$ is also the primary neurone responding to canna (Fig. 4A, traces 2 and 3 versus 6 and 7). As with the canna extract, responses from the lateral styloconica to the mixtures (Fig. 4B, traces 6 and 7) were more heterogeneous than those of the medial styloconica (Fig. 4A, traces 6 and 7). The average responses to the mixtures (Fig. 5C) were generally comparable with those elicited by their constituents at similar concentrations (Figs 5A, B).

Responses to caffeine. This well-known phagodeterrent for $M$. sexta was included here as a comparison with RA. Both chemicals elicited the same large spike, presumably from the same deterrent neurones (Fig. 4, traces 3 and 8). One difference between the effects of the two stimuli is that caffeine elicited a higher frequency of large spikes from the lateral than the medial styloconica, whereas responses to RA were similar in both styloconica (Fig. 5).

\section{Discussion}

The results of the present study strongly indicate that RA is an active principle in extracts of canna leaves. The calculated RA concentration found in the leaf is approximately $5 \mathrm{mM}$, which is five times the concentration that substantially inhibited feeding on it in the two-choice test. RA is a derivative of caffeic acid; some other derivatives of caffeic acid modulate the feeding of many species of lepidopteran larvae. For example, chlorogenic acid acts as a mild feeding deterrent to Spodoptera litura and is considered to play a role in the resistance of peanuts to this insect (Stevenson et al., 1993). It also enhances the antifeedant activity of Cicer isoflavonoids (Simmonds \& Stevenson, 2001). 


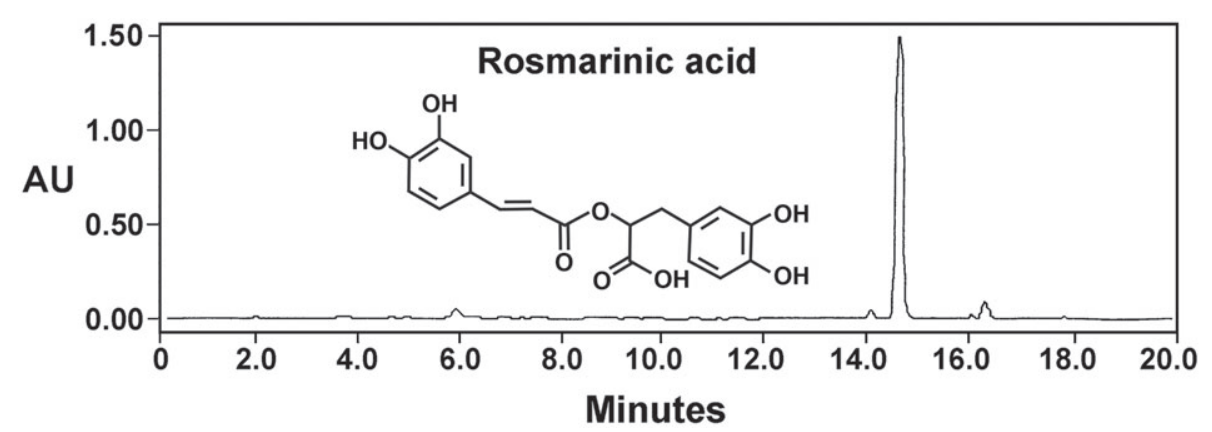

Fig. 1. High-performance liquid chromatography (HPLC) evidence that rosmarinic acid (RA) is in canna. HPLC trace at $310 \mathrm{~nm}$ of the $50 \%$ methanol eluted Sep-Pak fraction of the hot ethanol extract of foliage of canna. The large peak is identified as RA.

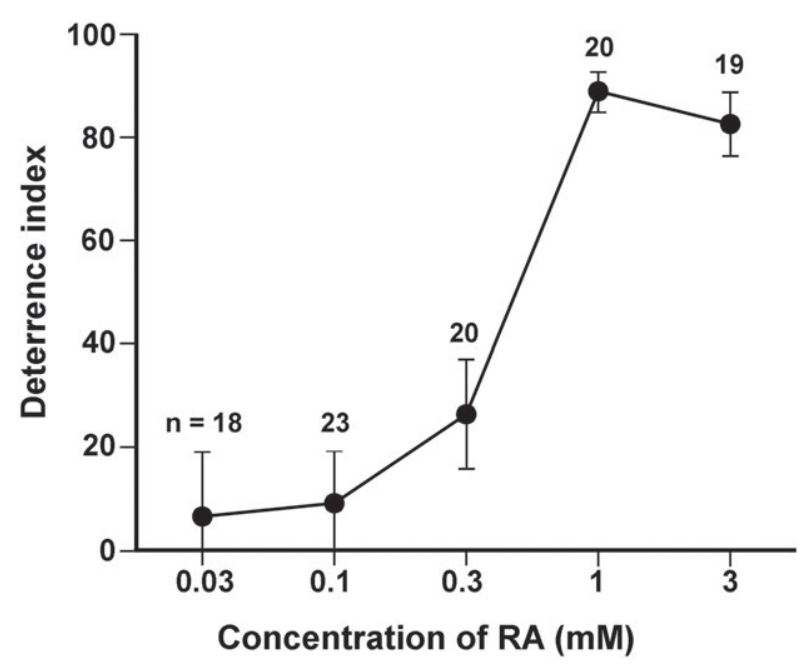

Fig. 2. Behavioural concentration-response curve to rosmarinic acid (RA). Symbols are the mean \pm SE of the Deterrence index for $n$ larvae in two-choice tests comprised of solutions of RA versus distilled water.

RA can be isolated from many species within the Boraginaceae and the subfamily Nepetoideae of the Lamiaceae; it can also be isolated from ferns belonging to the family Blechnaceae, as well as hornworts and species belonging to the sea grass families Zosteraceae and Potamogetonaceae (Peterson \& Simmonds, 2003). To our knowledge, none of the species known to contain RA are hosts of M. sexta. Further work should be undertaken to determine whether the medial styloconic sensillum is responsive to other caffeic acid derivatives. This could provide more information about the specificity of the neurone, as well as the role that this widely distributed group of compounds plays in the feeding behaviour of the hornworm.

Previous research on the effect of differential ablation of chemoreceptors on feeding behaviour shows that a single medial (but not lateral) styloconic sensillum alone is capable of blocking feeding on canna (de Boer \& Hanson, 1987). Similar ablation experiments in the present study using RA instead of canna show equivalent results: the medial (but not lateral) styloconica are important in rejection of RA. Therefore, the discovery that RA elicits a classic, single-deterrent neurone response in the medial sensillum is significant. In the $M$. sexta literature, this neurone

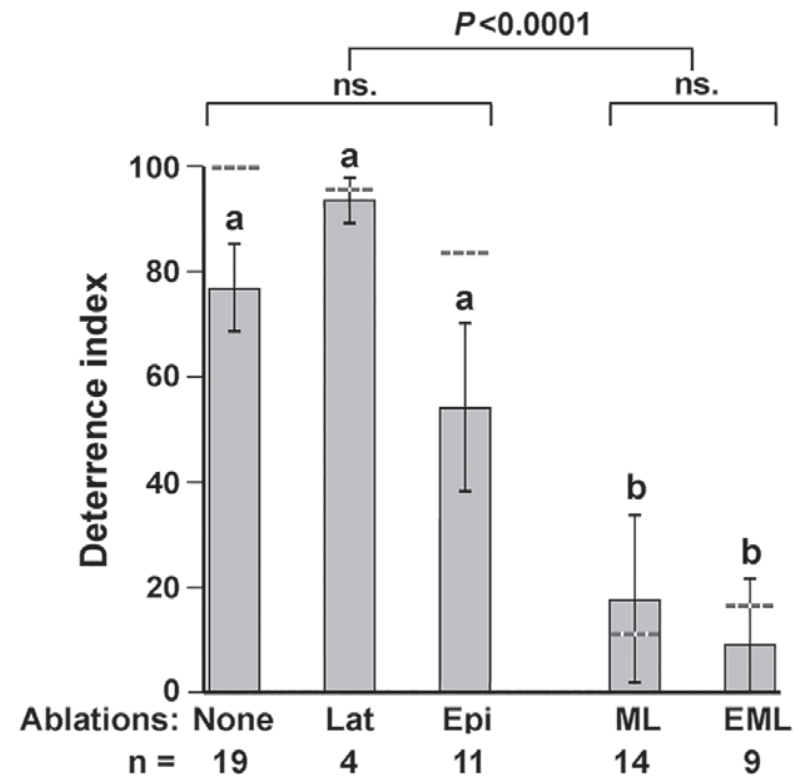

Fig. 3. Behavioural responses to rosmarinic acid (RA) by normal and chemosensory-deprived larvae. Control larvae were anaesthetized but not ablated (None). Bilateral ablation groups are: maxillary lateral styloconica (Lat); both lateral and medial styloconica (ML); epipharyngeal sensilla (Epi); and the combination of the epipharynx, medial and lateral styloconica (EML). Bars represent the mean $\pm \mathrm{SE}$, and dashed lines indicate the medians, of the Deterrence index for $\mathrm{n}$ larvae in 2-choice tests comprised of $1 \mathrm{~mm}$ RA versus distilled water. Lowercase letters denote significantly different groups (Kruskal-Wallace test, alpha $=0.05$ ); ns, not significant. Note that deterrence is essentially eliminated with loss of the medial styloconica (ML and EML).

is characterized by its response to partially purified fractions of canna leaves, and associated behavioural assays show that the activity of this neurone correlates well with phagodeterrence (Peterson et al., 1993). RA elicits almost identical responses in the present study, thus supporting the hypothesis that RA is the primary active principle in canna for deterring hornworm feeding. This exemplifies one generally accepted mechanism of deterrence, namely that rapid activity of a deterrent neurone blocks feeding. This and other mechanisms of deterrence are discussed by Bernays \& Chapman (2000) and Glendinning et al. 
1.

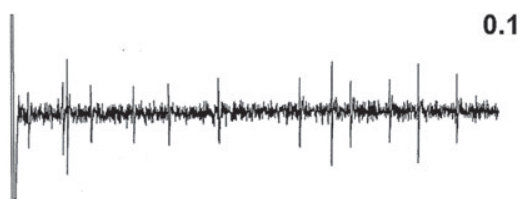

$0.1 \mathrm{M} \mathrm{KCl}$

2.

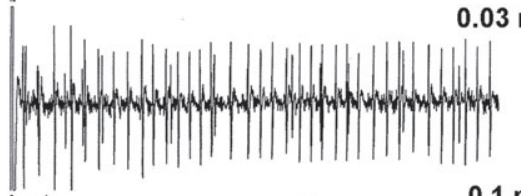

$0.03 \mathrm{mM}$ RA

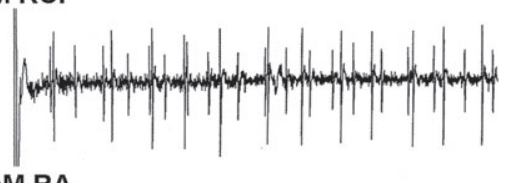

3.

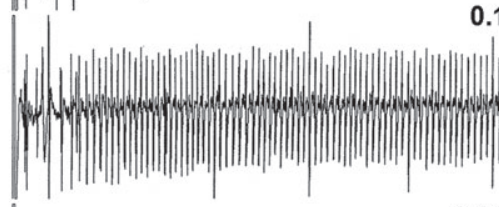

$0.1 \mathrm{mM}$ RA

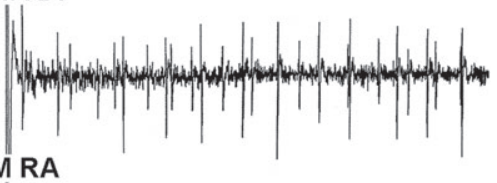

4.

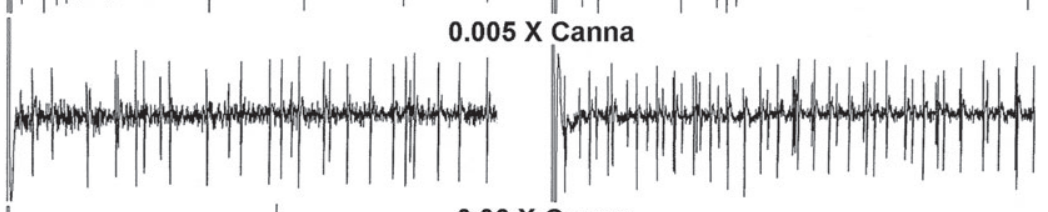

5.

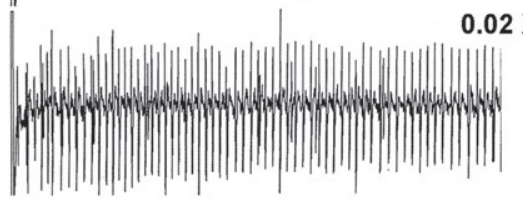

$0.02 \times$ Canna
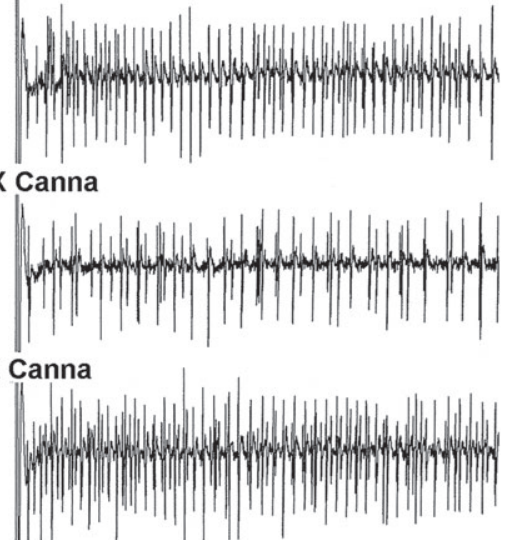

0.015 mM RA \& $0.0025 \times$ Canna

6.

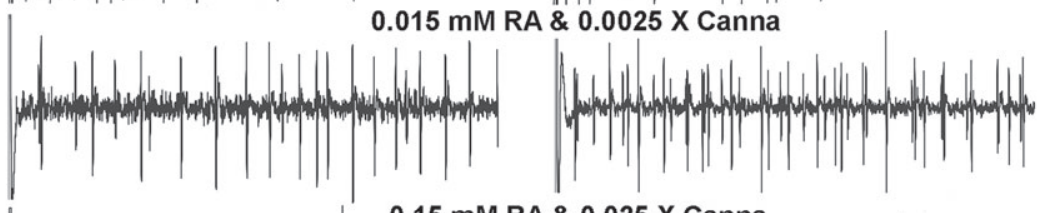

7.

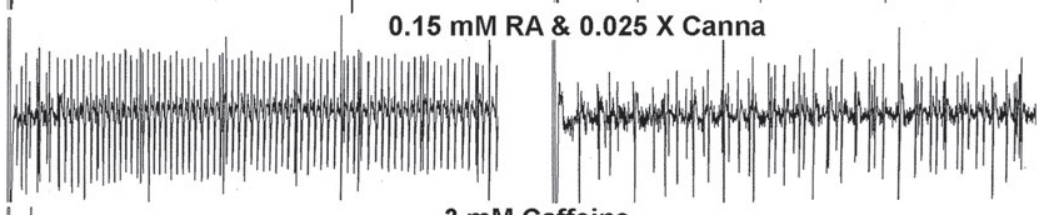

8.
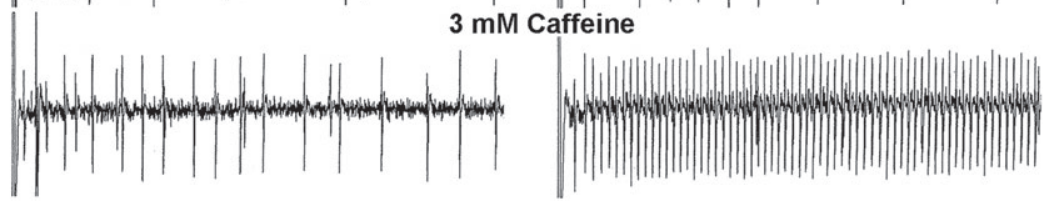

Fig. 4. Electrophysiological responses of the maxillary gustatory neurones to deterrents. (A) Medial styloconicum. (B) Lateral styloconicum. The duration of the traces shown is $600 \mathrm{~ms}$. starting from the initial contact artefact at the left of each trace. Canna concentrations listed are relative to full strength extract $(=1 \times)$. All solutions contained $0.1 \mathrm{M} \mathrm{KCl}$ as an electrolyte. All responses depicted are from the left maxilla of one animal. Note that the responses to rosmarinic acid (RA) are very similar to those of canna, especially in the medial styloconica.

(2002), and reviews are provided by Frazier (1986), Frazier \& Chyb (1995), Schoonhoven et al. (1992) and Schoonhoven \& van Loon (2002).

Although, in the present study, the lateral styloconica are shown to be quite responsive to both RA and canna, this sensillum does not appear to mediate deterrence to canna (de Boer \& Hanson, 1987), nor to RA (Fig. 3). Presumably, the multi-neuronal response to RA from the lateral sensillum is not a signal that is interpreted by the central nervous system as a phagodeterrent.
The potency of RA as a deterrent for the tobacco hornworm (behavioural threshold approximately $0.3 \mathrm{~mm}$ and electrophysiological threshold $<0.03 \mathrm{~mm}$ ) is comparable with that of many phytochemical deterrents tested in other insects (Schoonhoven \& van Loon, 2002). The phagodeterrence of RA varies from threshold to maximum in less than a decade of concentration range. This steep concentration-response curve is also characteristic of the responses of other insects to strong deterrents, such as the response of the locust Schistocerca gregaria to azadirachtin (Mordue (Luntz et al., 1998). 
(A)

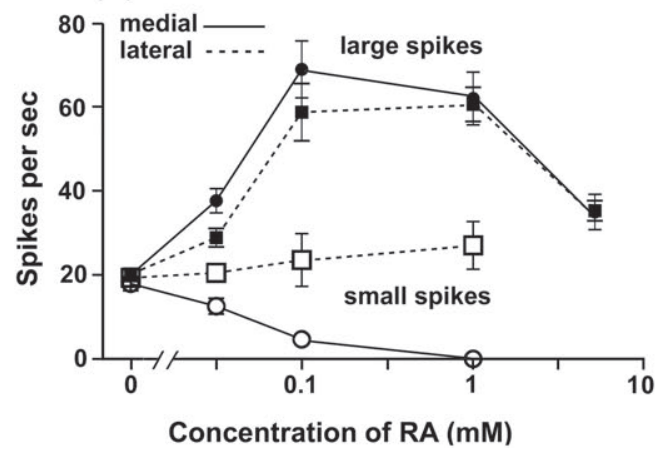

(B)

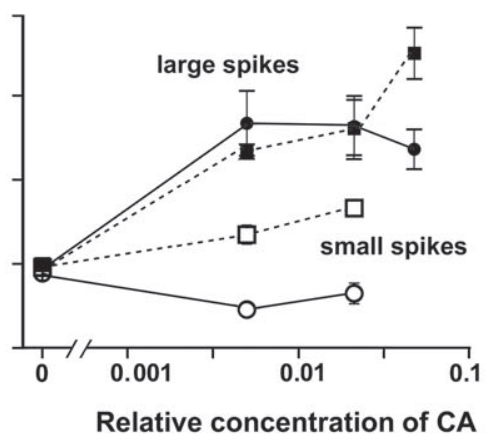

(C)

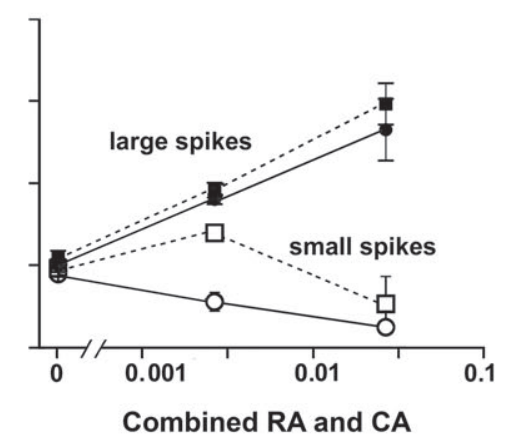

Fig. 5. Average concentration-response curves of the maxillary gustatory neurones to deterrents plotted on semi-log axes. (A) Rosmarinic acid (RA). (B) Canna extract (CA). Concentrations listed are relative to full strength extract $(=1 \times)$. (C) Mixtures of RA and CA, plotted as concentration of CA $(0.015 \mathrm{~mm}$ RA and $0.0025 \times \mathrm{CA}$ and $0.15 \mathrm{~mm} \mathrm{RA}$ and $0.025 \times \mathrm{CA})$. Each symbol represents the weighted average (mean $\pm \mathrm{SE})$ of the responses of six sensilla, each with two or three trials per concentration. Controls $(0.1 \mathrm{M} \mathrm{KCl}, \mathrm{RA}$ and CA concentration $=0)$ had nine or more trials. Solid lines denote medial styloconica; dashed lines denote lateral styloconica. Solid symbols represent large spikes; open symbols represent small spikes. Symbols without visible error bars represent data with an SE smaller than the symbol.

When a chemosensitive neurone is characterized as either a deterrent or phagostimulant neurone, the question arises as to whether it is a generalist or a specialist. The fact that only a small percentage of the total number of plant species is consumed by most phytophagous insects suggests that caterpillar deterrent neurones must be generalists because they are the gatekeepers of feeding that must exclude the vast majority of plants (Schoonhoven, 1982). To date, however, only two compounds, aristolochic acid (Glendinning et al., 2002) and now RA, are known to stimulate this 'RA neurone' in the medial styloconica of $M$. sexta, and only a few more deterrent stimuli (caffeine and salicin and related compounds, plus aristolochic acid) are known for the 'caffeine neurone' in the lateral styloconica (Schoonhoven, 1972; Glendinning et al., 1999; Cocco \& Glendinning, 2012). The responses to RA and caffeine in the present study (Fig. 4) exemplify the two known deterrent pathways, although presumably there are others as well. Their limitations, interactions and mechanisms of behavioural control are not well understood. For example, there are many phytochemicals that are deterrent for other species but not for $M$. sexta (Wrubel \& Bernays, 1990); it is not known whether these phytochemicals simply go undetected by the sensory neurones of M. sexta, or whether the hornworm's deterrent pathways are more permissive. Clearly, more information about deterrent compounds and their effects on insect sense organs and behaviour is needed to provide a better understanding of food selection mechanisms.

\section{Acknowledgements}

We gratefully acknowledge R. Grayer, Royal Botanic Gardens, Kew, for support with the phytochemical analyses. We also thank our colleagues at University of Maryland Baltimore County: C. Schwarz and M. Mogul, for their help with the experiments; K. Sawyer for data processing; T. Ford for figures; M. Rowley for software; and L. Stanwyck for statistics. We are also indebted to J. Stitt and J. Frazier of Penn State University for software and laboratory facilities, respectively, and to the anonymous reviewers for their insightful and constructive comments. The authors declare that they have no conflicts of interest.

\section{References}

Bernays, E.A. \& Chapman, R.F. (2000) A neurophysiological study of sensitivity to a feeding deterrent in two sister species of Heliothis with different diet breadths. Journal of Insect Physiology, 46, 905-912.

de Boer, G. \& Hanson, F.E. (1987) Differentiation of roles of chemosensory organs in food discrimination among hosts and non-host plants by larvae of the tobacco hornworm, Manduca sexta. Physiological Entomology, 12, 387-398.

Chapman, R.F. (1995) Chemosensory regulation of feeding. Regulatory Mechanisms in Insect Feeding (ed. by R.F. Chapman and G. de Boer), pp. 101-136. Chapman and Hall, New York, New York.

Cocco, N. \& Glendinning, J.I. (2012) Not all sugars are created equal: some mask aversive tastes better than others in an herbivorous insect. Journal of Experimental Biology, 215, 1412-1421.

Frazier, J.L. (1986) The perception of plant allelochemicals that inhibit feeding. Molecular Aspects of Insect-Plant Associations (ed. by L.B. Brattsten and S. Ahmad), pp. 1-42. Plenum Press, New York, New York.

Frazier, J.L. \& Hanson, F.E. (1986) Electrophysiological recording and analysis of insect chemosensory responses. Insect-Plant Interactions (ed. by J.R. Miller and T.A. Miller), pp. 285-330. Springer-Verlag, New York, New York.

Frazier, J.L. \& Chyb, S. (1995) Use of feeding inhibitors in insect control. Regulatory Mechanisms of Insect Feeding (ed. by R.F. Chapman and G. de Boer), pp. 364-381. Chapman and Hall, New York, New York.

Glendinning, J.I., Tarre, M. \& Asaoka, K. (1999) Contribution of different bitter-sensitive taste cells to feeding inhibition in a caterpillar (Manduca sexta). Behavioral Neuroscience, 113, 840-854.

Glendinning, J.I., Davis, A. \& Ramaswamy, S. (2002) Contribution of different taste cells and signaling pathways to the discrimination of 'bitter' taste stimuli by an insect. Journal of Neuroscience, 22, $7281-7287$. 
Gothilf, S. \& Hanson, F.E. (1994) A technique for electrophysiologically recording from chemosensory organs of intact caterpillars. Entomologia Experientia et Applicata, 72, 305-310.

Jermy, T., Hanson, F.E. \& Dethier, V.G. (1968) Induction of specific food preference in lepidopterous larvae. Entomologia Experientia et Applicata, 11, 211-230.

Mordue (Luntz), A.J., Simmonds, M.S.J., Ley, S.V. et al. (1998) Actions of azadirachtin, a plant allelochemical, against insects. Pesticide Science, 54, 277-284.

Peterson, M. \& Simmonds, M.S.J. (2003) Molecules of interest: rosmarinic acid. Phytochemistry, 62, 121-125.

Peterson, S.C., Hanson, F.E. \& Warthen, J.D. (1993) Deterrence coding by a larval Manduca chemosensory neurone mediating rejection of a non-hostplant, Canna generalis L. Physiological Entomology, 18, 285-295.

Schoonhoven, L.M. (1972) Secondary plant substances and insects. Structural and Functional Aspects of Phytochemistry (ed. by V.C. Runeckles and T.C. Tso), pp. 197-224. Academic Press, New York, New York.

Schoonhoven, L.M. (1982) Biological aspects of antifeedants. Entomologia Experientia et Applicata, 31, 57-69.

Schoonhoven, L.M. \& van Loon, J.J.A. (2002) An inventory of taste in caterpillars: each species its own key. Acta Zoologica Academiae Scientiarum Hungarica, 48, 215-263.

Schoonhoven, L.M., Blaney, W.M. \& Simmonds, M.S.J. (1992) Sensory coding of feeding deterrence in phytophagous insects. Insect-Plant
Interactions, Vol. 4 (ed. by E.A. Bernays), pp. 59-79. CRC Press, Boca Raton, Florida.

Simmonds, M.S.J. \& Stevenson, P.C. (2001) Effects of isoflavonoids from Cicer on larvae of Heliocoverpa armigera. Journal of Chemical Ecology, 27, 965-977.

Simpson, S.J. \& Raubenheimer, D. (1993) A multi-level analysis of feeding behaviour: the geometry of nutritional decisions. Philosophical Transactions of the Royal Society of London B, 342, 381-402.

Stevenson, P.C., Anderson, J.C., Blaney, W.M. \& Simmonds, M.S.J. (1993) Developmental inhibition of Spodoptera litura (Fab.) larvae by a novel caffeoylquinic acid from the wild groundnut, Arachis paraguariensis (Chod et Hassl.). Journal of Chemical Ecology, 19, 2917-2933.

Stitt, J.P., Gaumond, R.P., Frazier, J.L. \& Hanson, F.E. (1998) Action potential classifiers: a functional comparison of template matching, principal components analysis, and an artificial neural network. Chemical Senses, 23, 531-539.

Wrubel, R.P. \& Bernays, E.A. (1990) The relative insensitivity of Manduca sexta larvae to non-host plant secondary compounds. Entomologia Experientia et Applicata, 54, 117-124.

Yamamoto, R.T. (1969) Mass rearing of the tobacco hornworm. II. Larval rearing and pupation. Journal of Economic Entomology, 62, $1427-1431$.

Accepted 26 January 2019 